\title{
découpage par jets liquides en géotechnique
}

\author{
par
}

François Gilbert et Minh Phong Luong

Laboratoire de Mécanique des Solides C.N.R.S. - E. N.P.C. - E. N.S. M.P. - E. P.

\section{Introduction}

\subsection{Idée du découpage par jets liquides}

II est possible sous certaines conditions d'entamer un solide ou un assemblage de particules solides avec un fluide. Des exemples connus sont les effets destructeurs des vagues et des courants marins, l'érosion par les cours d'eau, les conséquences de la pluie à l'aplomb d'une gouttière percée ou les dommages dus à la cavitation dans les installations hydrauliques.

De ces observations découle le principe du découpage par jets liquides: on projette à grande vitesse un certain débit fluide sur une portion limitée d'un matériau. II se produit alors au voisinage de l'impact du jet un ou plusieurs phénomènes (poinçonnement, fracturation, arrachement...) dont les mécanismes exacts sont mal élucidés et qui conduisent à un découpage du matériau, à une perte de masse par érosion ou à la destruction par fragmentation.

On peut définir le jet comme une région de l'espace non limitée par des parois solides et d'étendue faible dans laquelle les vitesses fluides sont importantes. Le jet est créé à l'aide d'une installation hydraulique appropriée (pompe ou multiplicateur de pression) alimentée en énergie et en fluide (le plus souvent de l'eau pure ou avec quelques additifs). L'énergie de pression fournie au fluide par l'installation est transformée en énergie cinétique par un ajutage plus ou moins bien dessiné de faible diamètre, appelé buse, au travers duquel s'échappe le fluide.

On distingue diverses sortes de jets selon plusieurs critères :

a) La nature du milieu dans lequel se trouve le jet à la sortie de la buse : air ambiant (jet "libre") ou liquide (on parle alors de jet submergé).

b) L'allure du débit à la sortie de la buse au cours du temps, d'où la distinction entre jets continus (débit constant), modulés (débit variable) et pulsés (débit interrompu) (fig. 1).
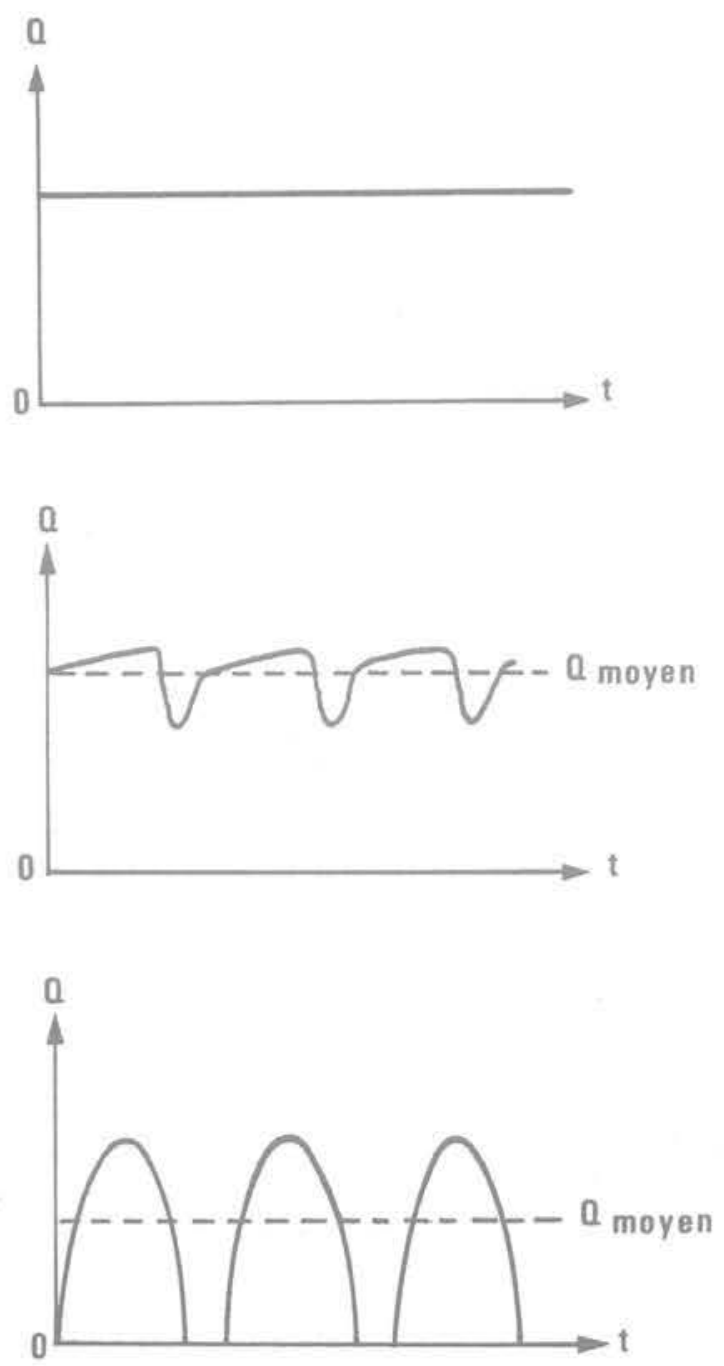

Fig. 1 Jet libre : débit à l'origine en fonction du temps pour un jet continu (a), modulé (b) ou pulsé (c) 
c) L'ordre de grandeur des vitesses à la sortie de la buse (position par rapport à la célérité du son) ou de la pression en amont de celle-ci. Pour fixer les idées disons qu'une pression de l'ordre de la dizaine de $\mathrm{MPa}$ (100 bar) correspond à peu près à une vitesse de $140 \mathrm{~m} / \mathrm{s}(500 \mathrm{~km} / \mathrm{h})$.

\subsection{Utilisation actuelle}

On utilise les jets liquides pour le découpage de divers matériaux, le nettoyage (décapage) ou l'assistance au découpage par des outils conventionnels. Certaines applications sont industrielles, d'autres au stade expérimental.

On découpe ainsi des mousses et d'autres matières plastiques, ainsi que le cuir et le caoutchouc (découpage des peaux et industrie de la chaussure en particulier), divers matériaux tissés et fibreux, dont le bois, le papier, le carton et la laine de verre, des produits alimentaires surgelés, la glace (dégagement de parois de quais en hiver), les sols, les roches, le béton et les métaux.

Naturellement les types de jets utilisés, les débits et les pressions à mettre en œuvre sont très différents selon les matériaux à découper (des pressions de quelques MPa suffisent pour creuser certains sols, il en faut plusieurs centaines pour découper un métal) et le résultat souhaité.

Le découpage par jets liquides peut être appliqué en géotechnique aux travaux suivants :

- découpage de tranchées,

- forage de trou par jet d'eau seul ou en assistance à un foret mécanique, pour le soutènement par boulonnage ou préalablement à un abattage par explosif.

- abattage sélectif de minerai,

- abattage hydraulique pur par « monitor i qui est une technique bien connue utilisée dans quelques mines de charbon dans le monde : on utilise dans ce cas des jets de gros diamètres ( 30 à $40 \mathrm{~mm}$ ) à des pressions faibles (quelques dizaines de $\mathrm{MPa}$ ),

- assistance à des pics ou des molettes en creusant grâce aux jets des saignées à proximité de l'outil mécanique dont les performances sont ainsi améliorées et l'usure réduite. Ceci peut être particulièrement intéressant pour les tunneliers,

- travaux d'ensouillage de canalisation : on pose d'abord la canalisation sur le fond marin puis on creuse la tranchée grâce à un traîneau équipé de buses qui se déplace sur la canalisation et creuse le sol sous elle.

- travaux de "débétonnage" de canalisations sousmarines,

- il existe enfin des projets d'exploitation hydraulique in-situ de petits gisements de certains minerais tendres: on amène par un sondage vertical jusqu'au minerai une buse animée d'un mouvement de rotation, qui brise le minerai; la pulpe ainsi formée serait remontée à la surface par pompage, décantée, triée et l'eau recyclée.

\subsection{Nécessité de modèles}

Du fait qu'il parait possible de découper à peu près n'importe quel matériau avec des jets liquides pourvu qu'ils soient assez rapides, on pourrait croire cet "outil» particulier appelé à remplacer beaucoup d'outils mécaniques conventionnels dans un proche avenir. II n'en est à l'évidence rien pour deux séries de raisons :

a) La technologie des pompes à très haute pression (plusieurs centaines de MPa) est encore mal maîtrisée. Ces machines sont chères et leur durée de vie est limitée en raison des conditions mécaniques extrêmes dans lesquelles elles fonctionnent. Des problèmes d'usure se posent également pour les buses et les joints tournants nécessaires pour certaines applications.

On peut sans doute compter sur les progrès techniques rapides dans ce domaine et le développement du marché pour améliorer la fiabilité des matériels et faire baisser leur prix dans les prochaines années.

b) L'utilisateur potentiel se heurte également aux problèmes suivants

- dans certains cas on n'arrive pas à obtenir le résultat souhaité sur un matériau : éclatement en plaquettes ou arrachement de blocs de formes diverses alors qu'on veut une saignée régulière. découpage trop lent ou trop peu profond...

- on ne sait pas vraiment prévoir quel type de jet est à utiliser sur un matériau donné et la puissance nécessaire pour la pompe lorsqu'on désire découper un matériau,

- enfin les mesures existantes d'énergie volumique de découpage (rapport de l'én̉ergie dépensée sur le volume déblayé) dite aussi énergie spécifique, donnent le plus souvent des chiffres très élevés par rapport à ceux obtenus avec des outils classiques.

Ceci fait apparaître les limites d'emploi des jets coupants et la nécessité de mieux comprendre les mécanismes en jeu lors du découpage par jets liquides.

A cette condition seulement on pourra prévoir avec une précision suffisante l'effet des jets sur un matériau, contrôler vraiment le phénomène et opérer dans les meilleures conditions en fonction du but poursuivi : découpage par des jets seuls ou amélioration des performances d'un outil assisté. L'objectif à atteindre sera souvent la minimisation des dépenses énergétiques totales: on cherchera ainsi les moyens de diminuer l'énergie volumique de découpage.

Il est donc indispensable de rassembler des résultats d'expériences suffisamment nombreux et comparables entre eux et de les analyser à l'aide de modèles théoriques recherchant une compréhension claire des mécanismes du découpage par jets.

La diversité des techniques employées (et dans certains cas leur complexité) impose de se cantonner dans un premier temps, pour arriver à cette compréhension, à un problème particulier choisi pour les simplifications introduites.

II s'agit du découpage d'une tranchée rectiligne de profondeur constante par un jet d'eau continu seul balayant à vitesse constante un matériau perméable cohérent et arrivant perpendiculairement sur celui-ci (fig. 2), le milieu ambiant étant l'air. Le matériau à découper est initialement sec.

II se trouve que ce type de problème est le plus simple qui se pose pour le découpage par jets liquides et qu'il présente en outre un intérêt pratique non négligeable par sa ressemblance avec divers problèmes "réels $n$.

On ne s'intéressera dans la suite qu'au découpage de sols et éventuellement de roches "tendres". 


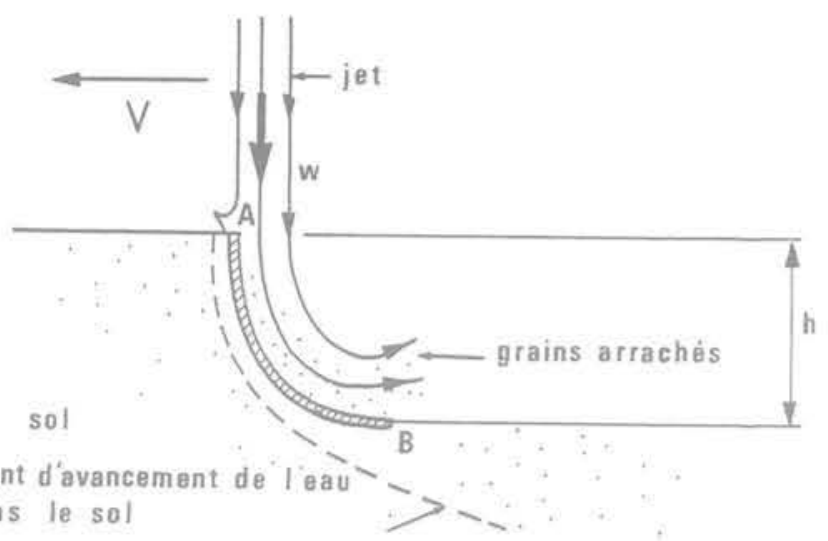

Fig. 2 Découpage d'une tranchée dans un sol initialement non saturé en eau : $w$ est la vitesse du jet et $V$ la vitesse de balayage; $A B$ est la surface de rupture et $h$ la profondeur de la tranchée

\section{Résultats expérimentaux sur le découpage des tranchées}

\subsection{Mode opératoire utilisé}

Soit une buse de géométrie donnée, de diamètre de sortie $D_{\mathrm{s}}$, placée à la distance $\mathrm{L}$ du sol et le balayant à une vitesse constante $\mathrm{V}$; on appelle $\mathrm{P}$ la pression juste en amont de la buse.
Le jet déblaye par unité de temps un volume de sol ' en creusant une tranchée de profondeur h constante: on peut définir la largeur $\ell$ de la tranchée (sans préjuger de la forme exacte de la tranchée) par l'égalitée : ' $\mathrm{I}=\mathrm{hV} \ell$, et la surface balayée $\mathrm{S}$ par unité de temps par: $\mathrm{S}=\mathrm{hV}=\frac{\mathrm{U}}{\mathrm{f}}$.

On a utilisé pour les expériences des jets à section circulaire (les seuls utilisables en pratique en raison des instabilités bien connues des autres sections et les seuls pour lesquels on trouve des buses dans le commerce) obtenus grâce à un multiplicateur dynamique de pression à double effet de rapport 59. La source basse pression était de l'air comprimé (on a utilisé un réseau fournissant de l'air pressurisé à environ $+0,7 \mathrm{MPa}$ ) et le liquide comprimé à forte pression (quelques dizaines de MPa) l'eau courante.

Le signal de pression juste en amont de la buse a été visualisé. grâce à un capteur, sur l'écran d'un oscilloscope numérique.

Les échantillons de sol à découper étaient placés sur un chariot mobile tiré à vitesse constante par un moteur électrique (mini-treuil). II était en effet plus simple de déplacer le sol que la buse, les échantillons ètant assez petits (cylindres de $75 \mathrm{~mm}$ de diamètre), quoiqu'encore grands devant les diamètres de sortie très faibles $(0,8 \mathrm{~mm}$ et $0,2 \mathrm{~mm})$ des buses utilisées. On a choisi en définitive une distance entre la buse et le sol pas trop grande et à peu près constante (environ $75 \mathrm{~mm}$ ) (fig. 3).

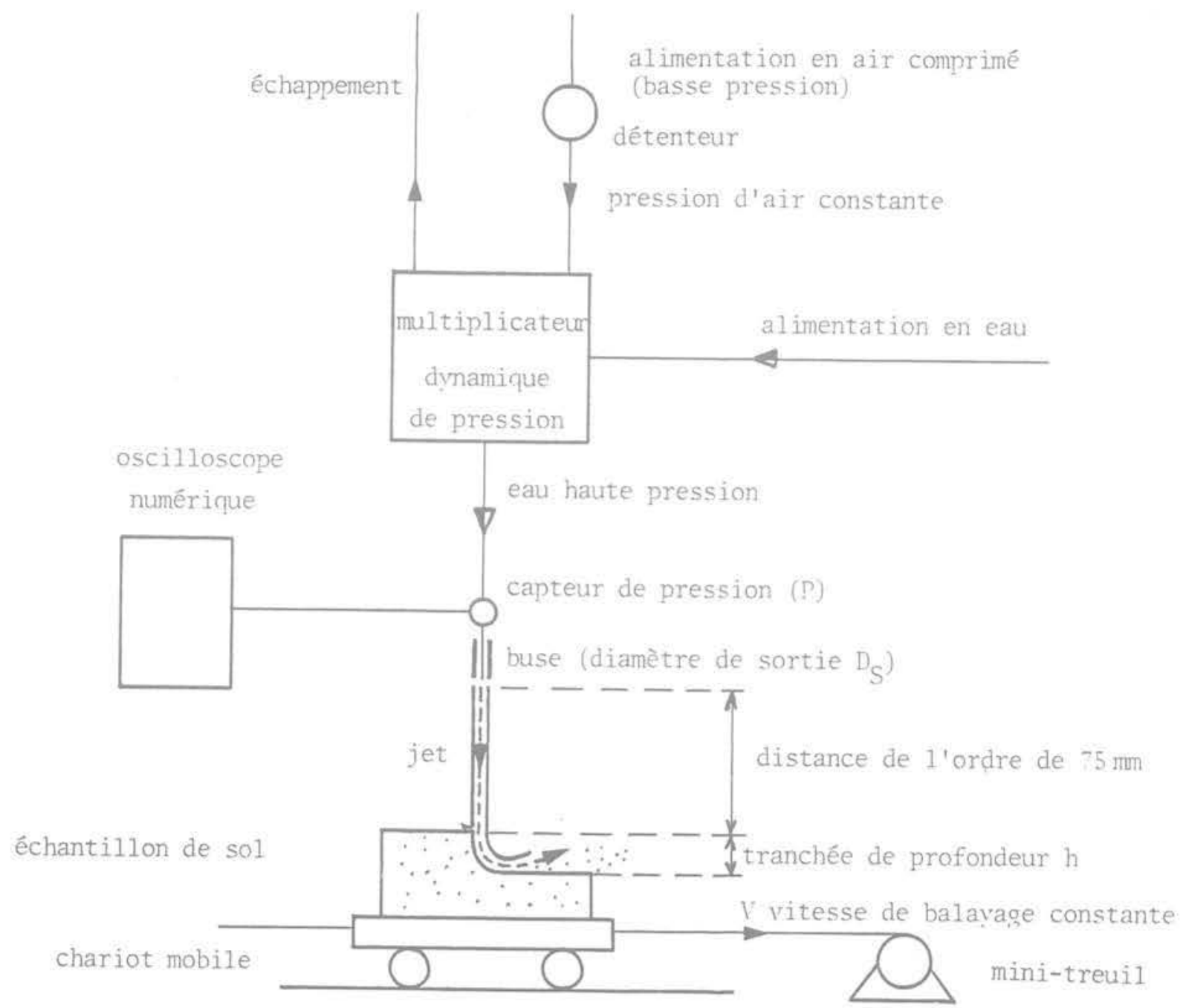

Fig. 3 Schéma de principe des expériences de découpage 


\subsection{Caractéristiques hydrauliques des jets}

Des considérations théoriques et expérimentales montrent qu'il existe une zone dans laquelle un jet ne diverge que très lentement et mettent en évidence pour le problème posé le rôle prépondérant joué par les deux nombres sans dimension suivants

- le coefficient de contraction m (inférieur à un), rapport de l'aire de la section contractée du jet à l'aire de la section de sortie de la buse; on sait en effet qu'il existe assez près de la sortie de la buse une section d'aire minimale oủ la vitesse w* est par conséquent maximale (conservation du débit):

$m=\left(\frac{D^{*}}{D_{\mathrm{s}}}\right)^{2}=\frac{\mathrm{w}_{\mathrm{s}}}{\mathrm{w}^{*}}<1$

$\left(D^{*}\right.$ diamètre de la section contractée, $D_{S}$ diamètre de sortie de la buse, $\mathrm{W}_{\mathrm{s}}$ vitesse moyenne à la sortie de la buse, $w^{*}$ vitesse moyenne dans la section contractée).

— un nombre d'Euler $\xi$ défini par:

$\xi=\frac{P}{P_{e} W^{* 2}}$.

( $P$ Pression en amont de la buse, $\rho_{e}$ masse volumique de l'eau), qui caractérise l'importance des pertes de charge; on montre en effet que $\xi \geqslant \frac{1}{2}$ et que lorsque les pertes de charge sont négligeables $\xi=\frac{1}{2}$.

On a mesuré pour les buses utilisées les valeurs prises par $m$ et $\xi$ grâce à des mesures de débit et de force exercée sur un obstacle. On montre en effet que :

$Q_{v}=\frac{\pi}{4} \cdot \frac{m}{\sqrt{\xi}} \cdot D_{s}^{2} \sqrt{\frac{P}{\rho_{0}}}$ (débit volumique)

$\mathrm{F}=\frac{\pi}{4} \cdot \frac{\mathrm{m}}{\xi} \cdot \mathrm{D}_{\mathrm{S}}^{2} \mathrm{P}$ (force exercée sur un obstacle)

d'oủ $m$ et $\xi$, les quantités $D_{S}, P$ et $\rho_{e}$ étant connues.

L'expérience a conduit à admettre que pour une buse donnée, le coefficient de contraction et le nombre d'Euler sont pratiquement constants dans une large gamme de nombre de Reynolds en régime turbulent.

La considération des valeurs prises par d'autres nombres sans dimension d'usage classique (nombres de Mach. Froude, Strouhal et Weber) a permis de conforter l'analyse précédente qui s'est révélée suffisante pour les problèmes de découpage.

La connaissance des nombres $m$ et $\xi$ permet en effet de calculer la vitesse moyenne $w$ dans une section du jet. le diamètre $D$ du jet, son débit volumique $Q_{v}=\frac{\pi}{4} D^{2} w$, la pression d'arrêt du jet $X$ donnée ici par $X=\rho_{e} \frac{W^{2}}{2}=\frac{P}{2 \xi}$ et sa puissance cinétique $s=Q_{\mathrm{y}} X$ (flux d'énergie cinétique traversant par unité de temps une section droite du jet), grandeurs d'intérêt technologique évident.

La puissance cinétique if est liée à l'énergie volumique de découpage $E_{S}$ (définie par rapport au jet) par la relation :

$E_{S}=\frac{f}{\partial}$

où ๆ est le volume déblayé par unité de temps.

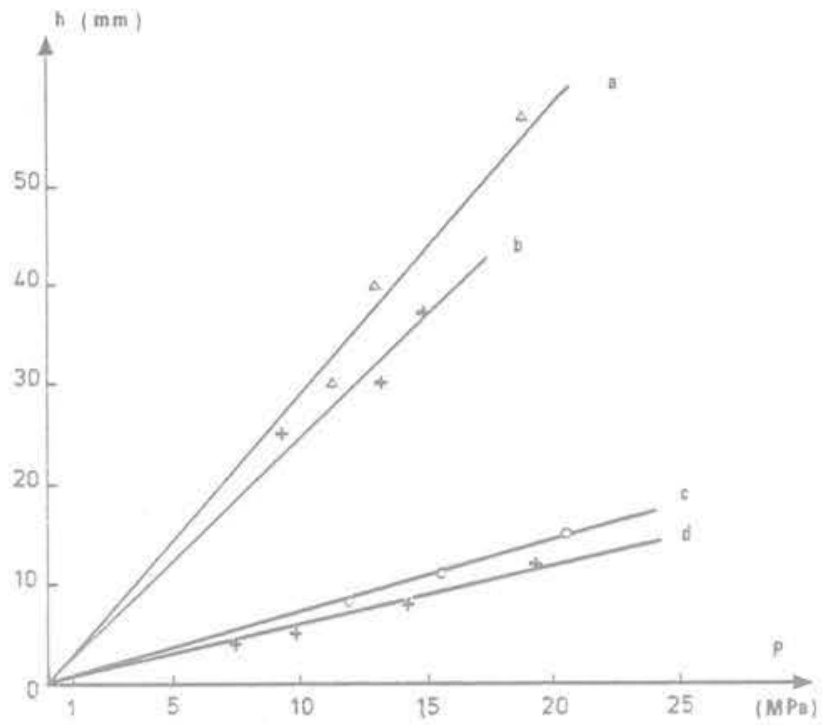

Fig. 4 Variations de la profondeur de la tranchée avec la pression (distance commune à toutes les expériences: environ $75 \mathrm{~mm}$ entre la buse et le sol)

a) argile :

$C=0,4 \cdot 10^{5} \mathrm{~Pa} \quad D_{S}=0,8 \mathrm{~mm} \quad V \cong 9 \cdot 10^{-3} \mathrm{~m} / \mathrm{s}$

b) argile :

$C=0,65 \cdot 10^{5} \mathrm{~Pa} \quad D_{S}=0,8 \mathrm{~mm} \quad \mathrm{~V} \cong 6 \cdot 10^{-3} \mathrm{~m} / \mathrm{s}$

c) marne argileuse :

$C=4 \cdot 10^{5} \mathrm{~Pa} \quad D_{S}=0,2 \mathrm{~mm} \quad V \cong 7.10^{-3} \mathrm{~m} / \mathrm{s}$

d) marne argileuse :

$C=4 \cdot 10^{5} \mathrm{~Pa} \quad D_{S}=0,2 \mathrm{~mm} \quad V \cong 10^{-2} \mathrm{~m} / \mathrm{s}$

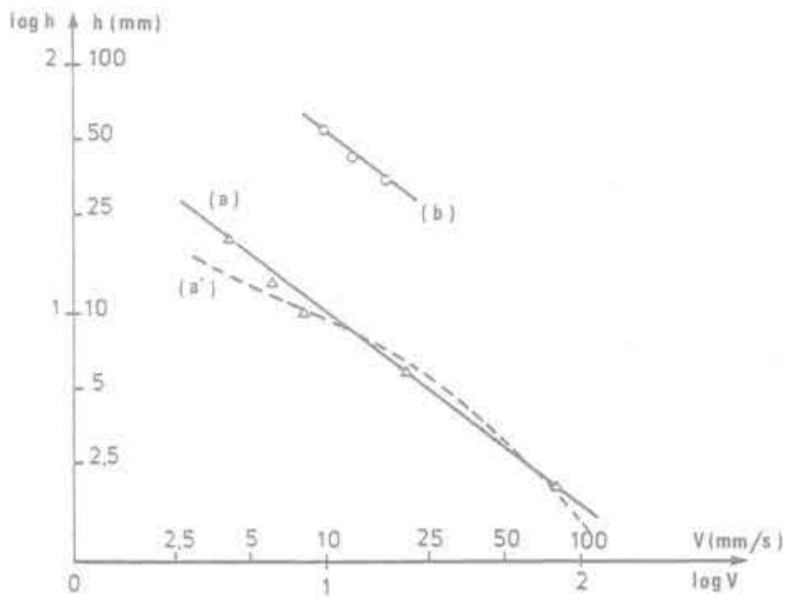

Fig. 5 Variations de la profondeur de la tranchée avec la vitesse de balayage

(a) marne argileuse

$C=7 \cdot 10^{5} \mathrm{~Pa}$, buse $\cdot D_{S}=0,8 \mathrm{~mm}$

$P \cong 23,5 \mathrm{MPa}$ (distance $80 \mathrm{~mm}$ )

(a') valeurs calculées de (a) avec:

$f=0,5 \quad b_{1}=6 \quad V_{1}=5 \cdot 10^{-3} \mathrm{~m} / \mathrm{s}$

(b) argile :

$C=0,6510^{5} \mathrm{~Pa}$, buse $D_{s}=0,2 \mathrm{~mm}$

$P \cong 17,5 \mathrm{MPa}$ (distance $79 \mathrm{~mm}$ )

Dans ces coordonnées bilogarithmiques, la pente de la courbe thérique est toujours (en valeur absolue) inférieure à un : c'est le cas ici pour les valeurs expérimentales 


\subsection{Résultats des essais}

Après avoir testé le comportement hydraulique des jets, on a procédé à des expériences de découpage de sols. On a mesuré, pour un sol donné et des valeurs de la pression et de la vitesse de balayage fixées, la profondeur $h$ de la tranchée dans la partie centrale de l'échantillon. On n'a pu évaluer avec l'installation utilisée ni le volume déblayé ni la largeur de la tranchée (trop faible pour être mesurée avec précision). On a montré que la profondeur de la tranchée ne dépendait pas de la distance buse-matériau choisie et on a alors tracé les variations de la profondeur $\mathrm{h}$ en fonction de la pression $\mathrm{P}$ et de la vitesse de balayage V (fig. 4 et 5).

On note que la profondeur est à peu près proportionnelle à la pression $\mathrm{P}$ et décroît assez rapidement quand la vitesse de balayage croît pour les deux matériaux utilisés : une marne argileuse et de l'argile de Provins consolidée.

Les limitations du montage empêchent d'obtenir des valeurs précises pour les conditions dites critiques définies ci-dessous, dont on sait d'ailleurs qu'elles sont peu nettes pour les sols.

Les résultats obtenus font apparaittre, en accord avec les observations qu'on peut trouver dans la bibliographie, les conclusions suivantes :

a) Pour une géométrie de buse donnée et aux distances $L$ pas trop grandes par rapport au diamètre de sortie de la buse $\mathrm{D}_{\mathrm{S}}$ la largeur de la tranchée semble à peu près proportionnelle à $\mathrm{D}_{\mathrm{s}}$.

b) La même remarque semble valable au moins en première approximation pour la profondeur $h$ de la tranchée.

c) L'efficacité du jet décroît lorsqu'on augmente la distance $L$ de la buse au matériau; cependant cet effet peut être tenu pour pratiquement négligeable jusqu'à des valeurs du rapport $L / D_{S}$ de l'ordre de plusieurs centaines. Dans les applications on se place le plus souvent assez près du matériau à découper et il n'est donc pas nécessaire de tenir compte de l'influence de la distance de la buse au matériau.

d) II existe une pression critique dépendant du matériau et de la vitesse de balayage au-dessous de laquelle le jet ne creuse pas ou très faiblement et irrégulièrement. Réciproquement il existe une vitesse de balayage critique dépendant du matériau et de la pression $\mathrm{P}$ au-dessus de laquelle on n'obtient pas de résultats de découpage intéressants. Ces limites sont plus ou moins nettes selon les cas.

e) On peut admettre en première approximation que la profondeur de la tranchée est proportionnelle à la pression P en amont de la buse lorsque celle-ci dépasse la pression critique.

f) Lorsqu'on fait varier la vitesse de balayage $V$ tout en restant au-dessous de la vitesse de balayage critique, on observe selon les cas trois types de comportement :

- soit la profondeur h de la tranchée semble à peu près constante (surface balayée proportionnelle à la vitesse de balayage),

- soit la surface balayée $S$ semble à peu près constante (profondeur de la tranchée inversement proportionnelle à la vitesse de balayage); si tel est le cas l'énergie volumique de découpage ne paraît pas dépendre de la cohésion du matériau,
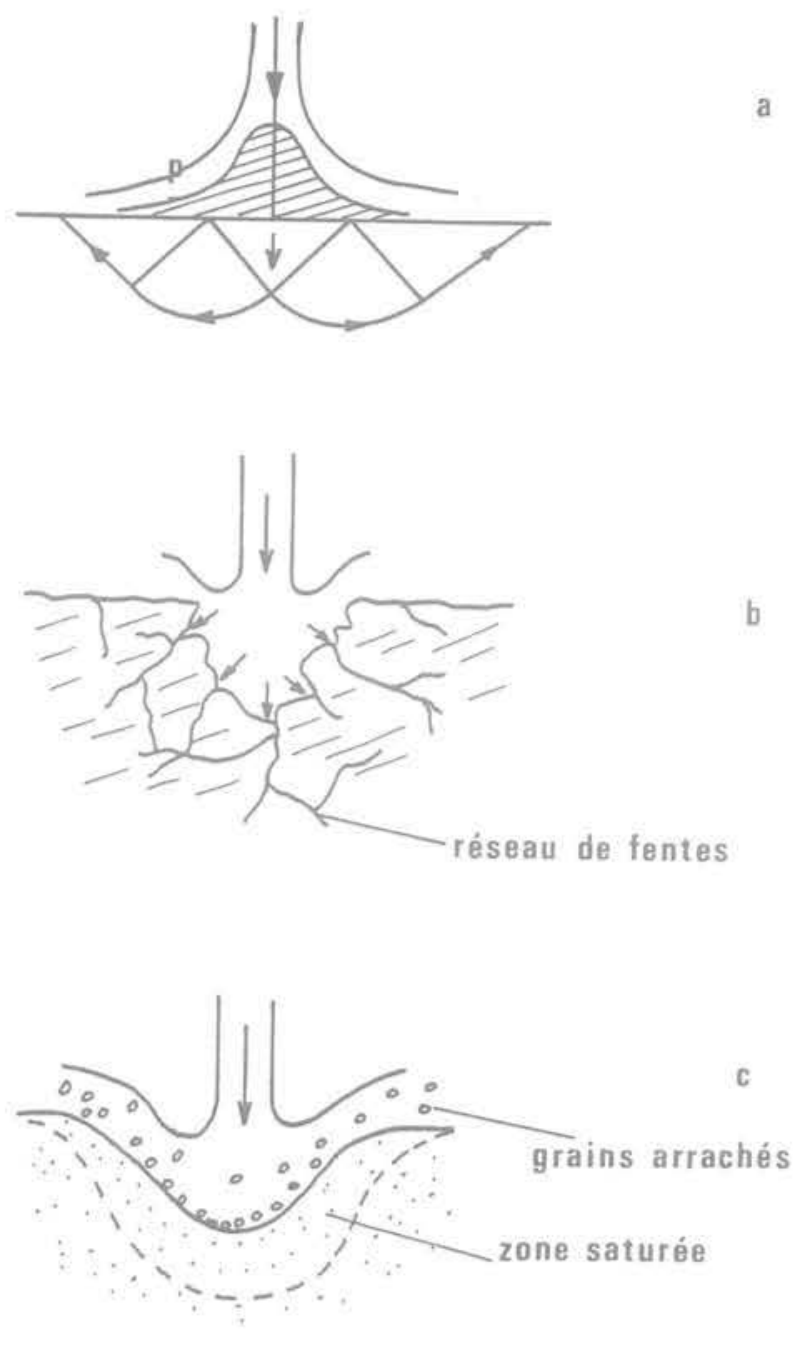

Fig. 6 Trois modes de découpage schématisés :

a) Poinçonnement en grandes déformations

b) Fracturation

c) Erosion

- soit on se trouve dans une situation intermédiaire : lorsqu'on fait croittre la vitesse de balayage $\mathrm{V}$ la profondeur de la tranchée décroît et la surface balayée par unité de temps croît; l'énergie volumique de découpage est alors une fonction strictement décroissante de la vitesse.

\section{Interprétation théorique}

\subsection{Analyse dimensionnelle}

II convient de préciser le type de mécanisme de découpage du sol envisagé. On peut en effet en distinguer au moins trois (fig. 6) :

a) Poinçonnement en grandes déformations : le jet repousse le matériau devant lui et l'écarte sans créer de débris;

b) Fracturation: le jet fait s'infiltrer de l'eau sous pression dans un réseau de fentes préexistantes et conduit à l'a éclatement " du matériau;

c) Érosion par arrachement : le jet exerce des efforts importants (pression et forces de frottement) sur les grains situés en surface et les arrache lorsqu'un certain critère de rupture est atteint; il y a en même temps infiltration. 
C'est ce dernier type de mécanisme qui doit très probablement être retenu pour les sols et certaines roches tendres à cause de la présence de débris de très faible dimension sans l'aspect caractéristique des matériaux fracturés.

Ceci étant la considération de l'effet de jets théoriques très larges (ceci pour s'affranchir du problème de la largeur exacte de la tranchée) se prête facilement à l'analyse dimensionnelle.

Sous réserve de négliger en particulier l'influence de la pesanteur et de la distance buse-matériau on montre qu'il doit exister une relation (dont la forme n'est pas connue a priori) entre la profondeur de la tranchée h/D rapportée à l'épaisseur du jet (ou à son diamètre dans le cas réel de jets à section circulaire) et les grandeurs sans dimension suivantes:

a) Le rapport $\frac{X}{C}$ où $X$ est la pression d'arrêt du jet et $C$ la cohésion effective du sol.

b) $\operatorname{tg} \varphi$, où $\varphi$ est l'angle de frottement interne effectif du sol.

c) Le coefficient de frottement équivalent $f$ à l'interface entre le jet et le sol, rapport en un point donné entre les efforts de cisaillement et de pression dus au jet.

d) Le rapport d/D oủ d est une taille caractéristique des grains du sol.

e) La porosité du sol $n$.

f) La quantité $k / d^{2}$ oủ $k$ est la perméabilité intrinsèque (supposée isotrope) du sol; cette quantité qui ne dépend que du sol considéré est d'ailleurs assez peu variable selon les sols si on prend pour taille caractéristique des grains le diamètre efficace de Allen Hazen et qu'on utilise pour déterminer la perméabilité $K=\frac{p_{e} g}{\eta} k$ la formule empirique de Hazen.

g) Un nombre de Reynolds de l'écoulement dans le sol $\mathrm{nV} \sqrt{\mathrm{k}} \rho_{\mathrm{e}} / \eta$ dans lequel $\mathrm{V}$ est la vitesse de balayage, $\rho_{e}$ la masse volumique de l'eau et $\eta$ sa viscosité dynamique.

h) Le groupement $\eta V d /(k C)$ dont le rôle apparaîtra par la suite dans la description du phénomène.

Pour aller plus loin, c'est-à-dire pour préciser la forme de la relation entre ces diverses grandeurs, il faut en pratique faire un certain nombre d'hypothèses simplificatrices, poser les équations et les résoudre, au moins de manière approchée.

\subsection{Modélisation effectuée}

La modélisation effectuée repose essentiellement sur les hypothèses suivantes

- on écrit que la pression moyenne sur la surface de découpage est due à la courbure des lignes de courant moyennes (influence des forces centrifuges) et que chaque grain situé à la surface du sol subit une traînée proportionnelle à la pression moyenne sur le fond, ce qui donne un coefficient de frottement équivalent à l'interface constant;

- on suppose les grains petits devant l'épaisseur du jet, ce qui est généralement bien vérifié;

- on admet que le critère de rupture (critère de Coulomb en contraintes effectives) est atteint sous la première couche de grains et que la pression interstitielle peut se calculer à l'aide de la loi de Darcy.
Ceci conduit pour les jets à section circulaire à la formule :

$\mathrm{h}=2 \mathrm{~b}, \mathrm{D} \frac{\mathrm{X}}{\mathrm{C}} \mathcal{H}\left(\mathrm{f}, \frac{\mathrm{V}}{\mathrm{V}_{\mathrm{i}}}\right)$

dans laquelle apparaissent un facteur de forme constant $b_{1}$ traduisant empiriquement le passage de deux à trois dimensions et une fonction $J$ sans dimension de deux variables qui se présente sous la forme d'une intégrale:

$\mathcal{H}\left(\mathrm{f}, \frac{\mathrm{V}}{\mathrm{V}_{1}}\right)=f \mathrm{e}^{-\mathrm{t} / 2} \int_{0}^{\pi / 2} \frac{\sin \lambda e^{t \lambda} \mathrm{d} \lambda}{1+\frac{V}{V_{1}} \sin \lambda}$

On appelle profondeur adimensionnelle cette quantité J que l'on peut tabuler; elle dépend du coefficient de frottement équivalent $f$ à l'interface entre le jet et le sol et du rapport de la vitesse de balayage $V$ à une quantité $V_{1}$, dite vitesse intrinsèque, qui a les dimensions d'une vitesse et est donnée sous les hypothèses faites par :

$V_{1}=\frac{k C}{\eta n d t g}$.

On vérifie aisément que la formule donnant la profondeur de la tranchée est de la forme générale précédente. Elle relie en effet h/D à $X / C$, $f$ et au groupement produit :

$\frac{\eta \mathrm{Vd}}{\mathrm{kC}} \cdot \mathrm{n} \cdot(\operatorname{tg} \varphi)=\frac{\mathrm{V}}{\mathrm{V}_{1}}$

et les hypothèses faites conduisent à négliger l'influence du nombre de Reynolds de l'écoulement dans le sol, du terme peu variable $\mathrm{k} / \mathrm{d}^{2}$ et du rapport d/D supposé très petit devant un.

Le modèle fournit en outre les conditions critiques et la forme théorique du front de découpage (assez proche d'un arc de spirale logarithmique) et permet de satisfaire aux conditions énoncées au paragraphe 2.3 (fig. 7 et 8 ).

La position de la vitesse de balayage par rapport à la vitesse intrinsèque traduit la plus ou moins grande facilité du découpage. Pour les variations de la profondeur de la tranchée et de la surface balayée par unité de temps on peut utilement introduire à côté de la profondeur adimensionnelle $\mathfrak{H}$ une surface balayée adimensionnelle $\delta$ par :

$S\left(f, \frac{V}{V_{1}}\right)=\frac{V}{V_{1}} \mathcal{H}\left(f, \frac{V}{V_{1}}\right)$

On voit alors apparaître très simplement les trois types de comportement avec la vitesse de balayage signalés au paragraphe 2.1, qui correspondent pour le modèle à des ordres de grandeur différents pour la vitesse intrinsèque du sol (fig. 9) :

- $V_{1}$ très grande par rapport aux vitesses de balayage employées : la profondeur de la tranchée semble à peu près indépendante de la vitesse de balayage,

- $V_{1}$ très petite : la surface balayée est à peu près constante et indépendante de la cohésion du matériau (ce qui a fait croire à certains qu'il fallait prendre en compte des propriétés nouvelles autres que la cohésion pour expliquer le découpage par jets liquides; on voit ici que ce n'est pas nécessaire),

- $V_{1}$ moyenne : c'est le cas intermédiaire oủ lorsqu'on fait croître la vitesse de balayage on observe effectivement une diminution de la profondeur de la tranchée et une augmentation de la surface balayée. 


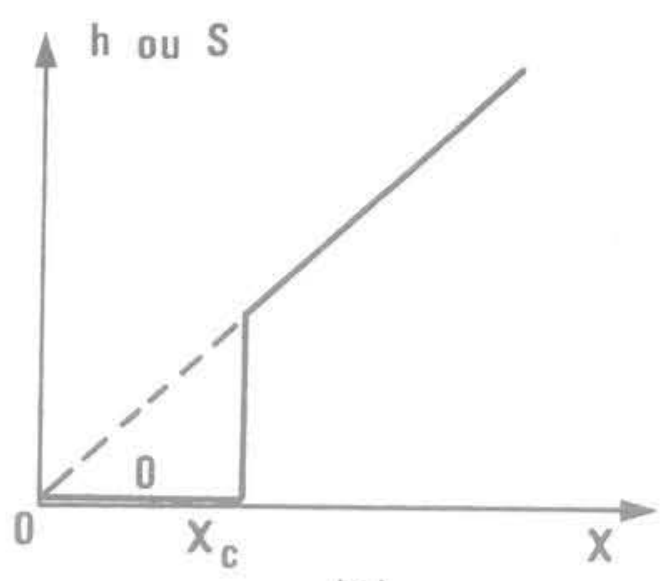

(a)

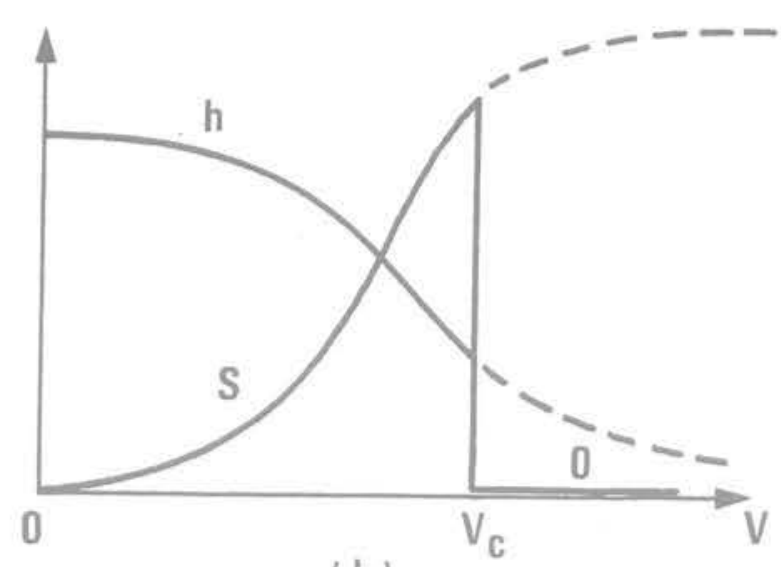

(b)

Fig. 7 Profondeur h de la tranchée obtenue et surface balayée par unité de temps $S$ pour un sol et une buse donnée : a) à vitesse de balayage $V$ fixée et pression d'arret du jet $X$ variable

b) à pression d'arrêt du jet fixée et vitesse de balayage variable

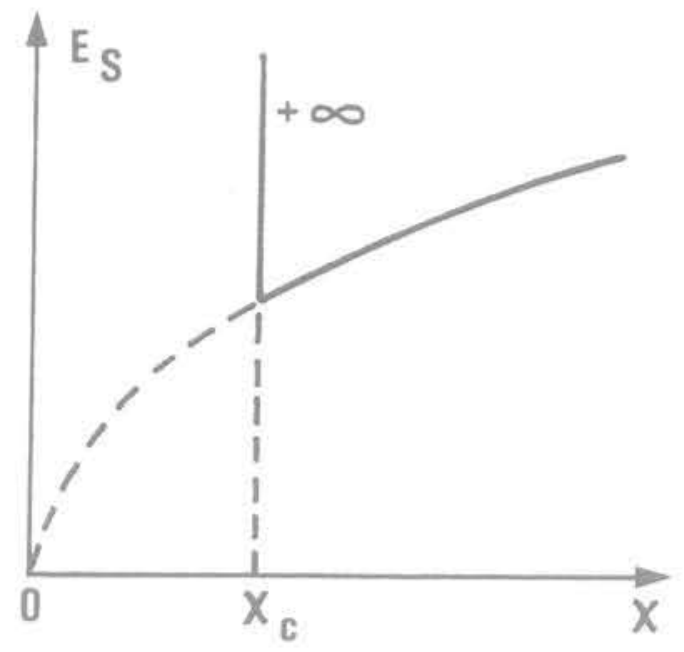

(a)

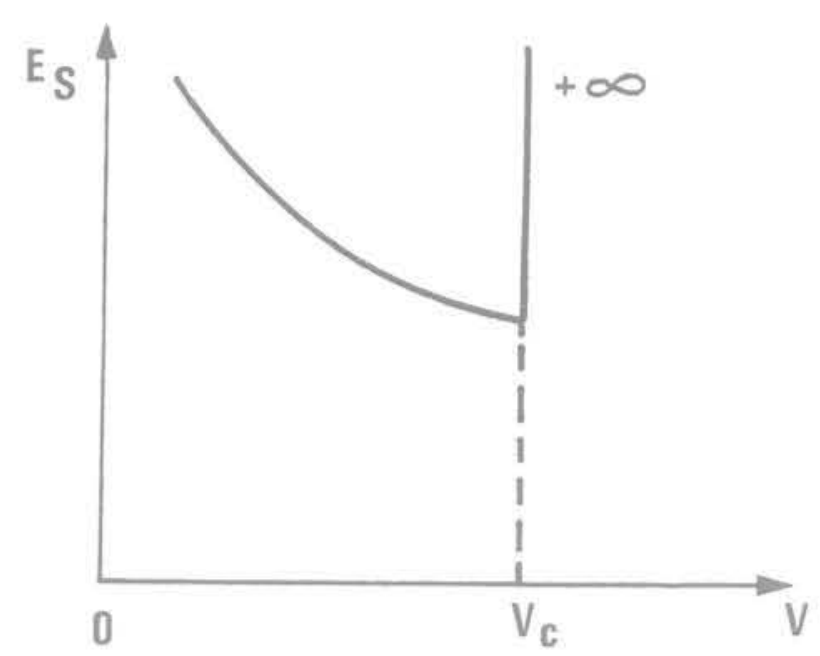

(b)

Fig. 8 Energie volumique de découpage d'un sol donné $E_{S}$; les cas a) et b) correspondent à ceux de la figure précédente

\subsection{Formules pratiques}

En tenant compte des caractéristiques de la buse et du jet, on écrit finalement que :

$\mathrm{h}=\mathrm{b}_{1} \frac{\sqrt{\mathrm{m}}}{\xi} \mathrm{D}_{\mathrm{s}} \frac{\mathrm{P}}{\mathrm{C}} \mathcal{H}\left(\mathrm{f}, \frac{\mathrm{V}}{\mathrm{V}_{1}}\right)$

$\ell=\mathrm{b}_{2} \sqrt{\mathrm{m}} \mathrm{D}_{\mathrm{S}}$

$S=b_{1} \frac{\sqrt{m}}{\xi} D_{s} \frac{P}{C} V_{1} s\left(f, \frac{V}{V_{1}}\right)$

$E s=\frac{\pi}{8 b, b_{2}} \frac{C}{V_{1}} \sqrt{\frac{P}{p_{e} \xi}} \frac{1}{\delta\left(f, \frac{V}{V_{1}}\right)}$

où $S\left(f, \frac{V}{V_{1}}\right)=\frac{V}{V_{1}} H\left(f, \frac{V}{V_{1}}\right)$ et $V_{1}$ est la vitesse intrinsèque, ces formules étant valables au-dessous de la vitesse de balayage critique $\mathrm{V}_{\mathrm{c}}$ :

$\mathrm{V}_{\mathrm{c}} \# \mathrm{~V}_{1}\left(\frac{\mathrm{Pf}}{2 \xi \mathrm{C}}-1\right)$
Les coefficients $b_{1}$ et $b_{2}$ sont des constantes de lordre de quelques unités, le diamètre de sortie de la buse $D_{S}$ et la pression en amont de la buse P sont connus, les quantités get $m$ peuvent être aisément mesurées et $\rho_{0}$ vaut $10^{3} \mathrm{kgm}^{-3}$. II reste donc à estimer la cohésion effective $C$, la vitesse intrinsèque $V_{1}$, pour laquelle on $a$ une formule theorique, et le coefficient de frottement équivalent $f$ dont l'influence est assez faible ce qui permet de se fixer une valeur a priori.

On note l'intérêt de réaliser un écoulement de «bonne qualité $n$ ( $\xi$ faible et $m$ élevé) et que la cohésion intervient également dans le terme $V_{1}$ qu'on peut estimer expérimentalement.

II est intéressant d'étudier l'influence sur les résultats du modèle des divers paramètres utilisés.

Ceci peut être résumé pour l'essentiel par le tableau suivant où le signe + indique une fonction croissante du paramètre considéré, le signe - une fonction 
décroissante et 0 l'absence d'influence; chaque colonne correspond à un paramètre et chaque ligne à une grandeur résultante d'intérêt technologique.

\begin{tabular}{c|c|c|c|c|c|c|c|c|c} 
& $\mathrm{m}$ & $\xi$ & $\mathrm{D}_{\mathrm{s}}$ & $\mathrm{P}$ & $\mathrm{V}$ & $\mathrm{C}$ & $\varphi$ & $\frac{k}{\mathrm{n}}$ & $\mathrm{d}$ \\
\hline $\mathrm{V}_{\mathrm{i}}$ & 0 & 0 & 0 & 0 & 0 & + & - & + & - \\
\hline $\mathrm{V}_{\mathrm{c}}$ & 0 & - & 0 & + & 0 & - & - & + & - \\
\hline $\mathrm{h}$ & + & - & + & + & - & - & - & + & - \\
\hline $\mathrm{S}$ ou $v$ & + & - & + & + & + & - & - & + & - \\
\hline $\mathrm{E}_{\mathrm{s}}$ & 0 & - & 0 & + & - & + & + & - & + \\
\hline
\end{tabular}

Outre la remarque déjà faite sur l'intérêt de réaliser un écoulement avec $\xi$ faible et $m$ élevé, il convient de noter que le modèle prévoit qu'un sol sera d'autant plus facilement découpé qu'il sera plus perméable ou constitué de grains fins comme on le lit sur les deux dernières colonnes du tableau. (Dans la réalité un sol fin est très peu perméable et l'influence de la perméabilité est largement prépondérante!) On vérifie aussi sur le tableau que la profondeur de la tranchée sera d'autant plus faible que le sol aura des caractéristiques mécaniques élevées ( $C$ et $\varphi$ importants).

\section{Conclusion}

Ce type de modèle, quoique simpliste, fournit quatre sortes de renseignements utiles pour les applications à la géotechnique :

a) II donne des ordres de grandeur pratiques permettant de se prononcer dans beaucoup de cas sur la faisabilité du découpage par jets liquides et son intérêt économique (prévision des débits et des pressions à mettre en cuvre, dépense énergétique...).

b) II permet de raisonner à partir d'un cadre, ce qui est indispensable pour appréhender les mécanismes en jeu et mieux maîtriser les techniques de découpage par jets liquides. Les prévisions, même si elles sont souvent plus qualitatives que vraiment quantitatives, sont un guide précieux dans un domaine encore mal connu.

c) II clarifie le rôle de la vitesse de balayage V, dont l'influence paraît souvent déroutante. II indique en effet que les performances de découpage dépendent de manière essentielle du rapport de cette vitesse à une vitesse intrinsèque dont il importe en conséquence de déterminer l'ordre de grandeur, soit expérimentalement soit à l'aide de formules telles que celle proposée. II est en particulier vain de chercher à établir des corrélations entre divers matériaux en ne prenant en compte que la vitesse de balayage elle-même, comme on cherche souvent à le faire.

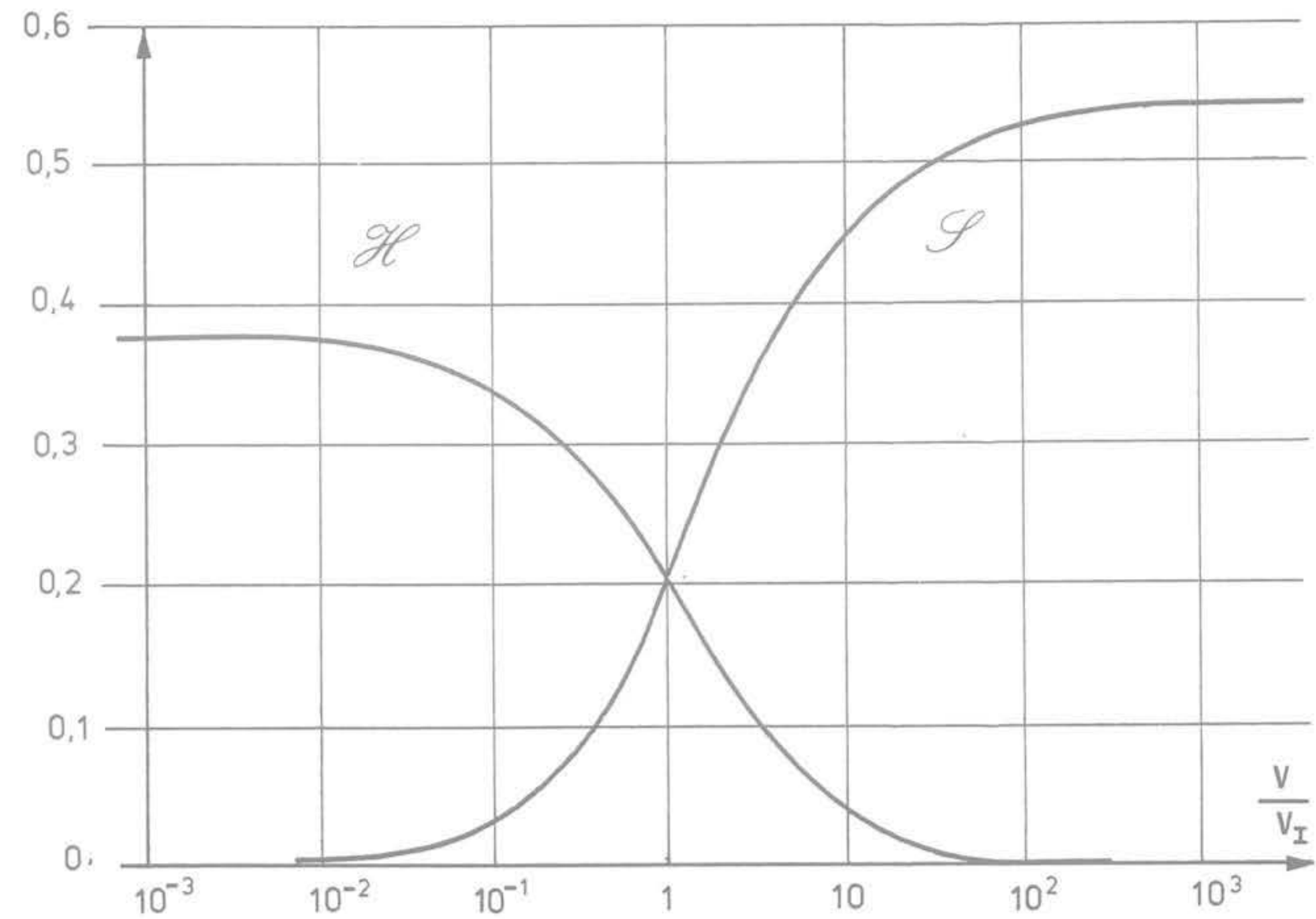

Fig. 9 Profondeur adimensionnelle $\mathfrak{T}$ et surface balayée adimensionnelle en fonction de la vitesse de balayage adimensionnelle $V / V_{1}$ (pour $\left.f=0,5\right)$ 
d) II montre enfin qu'en adoptant comme critère d'efficacité l'énergie volumique de découpage il est avantageux d'opérer à basse pression et vitesse de balayage élevée, quitte à faire plusieurs passes pour atteindre la profondeur souhaitée (ce n'était pas évident a priori). Ceci étant les conditions critiques apparaissent alors comme des conditions limites au voisinage desquelles on obtient une efficacité maximale en minimisant l'énergie volumique de découpage.

\section{Notations}

$b_{1}, b_{2}$ constantes numériques

C cohésion effective

d taille caractéristique des grains

D diamètre du jet

$D_{s}$ diamètre de sortie de la buse

$D^{*} \quad$ diamètre de la section contractée du jet

$\xi$ nombre d'Euler de la buse

$E_{S}$ énergie volumique de découpage

$f$ coefficient de frottement équivalent à l'interface entre le jet et le sol

g accélération de la pesanteur

h profondeur de la tranchée

I profondeur adimensionnelle

$k$ perméabilité intrinsèque $\left(\mathrm{m}^{2}\right)$

K perméabilité $(\mathrm{m} / \mathrm{s})$

$\ell \quad$ largeur de la tranchée

$\mathrm{L} \quad$ distance buse-matériau

m coefficient de contraction

n porosité du sol

T puissance cinétique du jet

P pression en amont de la buse

$\mathrm{Q}_{\mathrm{V}}$ débit volumique du jet

S surface balayée adimensionnelle

S surface balayée par unité de temps

ข volume déblayé par unité de temps

$\checkmark \quad$ vitesse de balayage

$V_{C} \quad$ vitesse critique de balayage

$V_{1} \quad$ vitesse intrinsèque

w vitesse moyenne du jet $\mathrm{w}_{\mathrm{s}} \quad$ vitesse moyenne à la sortie de la buse

$w^{*} \quad$ vitesse moyenne dans la section contractée

$\mathrm{X}$ pression d'arrêt du jet

$\eta \quad$ viscosité dynamique de l'eau

$\varphi \quad$ angle de frottement interne effectif

$\mathrm{P}_{\mathrm{e}} \quad$ masse volumique de l'eau

\section{Références bibliographiques}

S. C. Crow (1973) "A theory of Hydraulic Rock Cutting «, Int. J, Rock Mech. Min. Sci. et Geomech. Abstr., Vol $10 n^{\circ} 6$, pages 567-584.

C. Dereix, J. Gril, H. Moumadi (1981) "Un Outil Nouveau: Le Jet d'Eau à Haute Pression ". École Nationale du Génie Rural, des Eaux et des Forêts, microthème, Paris, décembre 1981

F. Gilbert (1981) "Modélisation du Découpage d'un Sol par Jets Liquides sous Forte Pression", Thèse présentée à l'École Nationale des Ponts et Chaussées. Paris, soutenue le 10 juin 1981, pour obtenir le grade de Docteur-Ingénieur.

Hamel (1981) “Les Jets d'Eau Haute Pression appliqués à l'Exploitation Minière". École Nationale Supérieure des Mines de Paris, Centre de Mécanique des Roches, Fontainebleau.

Proceedings of the Workshop on the Application of High Pressure Water Jet Cutting Technology, 1975, University of Missouri-Rolla.

Proceedings of the First International Symposium on Jet Cutting Technology, Coventry, 1972.

Proceedings of the Second International Symposium on Jet Cutting Technology, Cambridge, 1974.

Proceedings of the Third International Symposium on Jet Cutting Technology, Chicago, 1976.

Proceedings of the Fourth International Symposium on Jet Cutting Technology, University of Kent, Canterbury, 1978.

Proceedings of the Fifth International Symposium on Jet Cutting Technology, Hanover, 1980. 
